\title{
Mobility transition of older adult populations in Worcester, South Africa
}

\author{
* Catharina Maria Elizabeth Duvenage; M Augmentative and Alternative Communication (UP), \\ B. Occupational Therapy (UFS). https://orcid.org/0000-0003-2524-0249 \\ Occupational Therapist, Ukwanda Centre for Rural Health, Worcester, South Africa
}

\author{
Lizette Swanepoel; B Radiography (UP), B Occupational Therapy (UP), M Occupational Therapy (SU). \\ https://orcid.org/0000-0002-6662-707 I \\ Occupational Therapist in Private Practice CBS and Partners: Work Rehab, Somerset West, South Africa
}

\author{
\#Kopane Rehaona Dube; BSc. Human Life Science (SU), BSc. (Hons) Medical Virology (SU), \\ MSc. Medical Physiology (SU). https://orcid.org/0000-0002-6609-35 I 6 \\ Research Intern, Stellenbosch University, Ukwanda Centre for Rural Health, Worcester, South Africa
}

\#Rheta-Maria Smith; B.Occupational Therapy (SU). https://orcid.org/0000-0003-2435-4535

Occupational Therapist, Potchefstroom Hospital, Potchefstroom, South Africa

\#Tamsin Jean Ballantyne; B.Occupational Therapy (SU). https://orcid.org/0000-0002-2793-8002

Occupational Therapist, Mokopane Regional Hospital, Limpopo, South Africa

\#Isolde Burger; B.Occupational Therapy (SU). https://orcid.org/0000-0003-304 I-5680.

Occupational Therapist, Potchefstroom Hospital, Potchefstroom, South Africa.

\section{\#Asisipho Tsotsa; B.Occupational Therapy (SU). https://orcid.org/0000-0002-8689-7095}

Occupational Therapist, Tower Hospital, Fort Beaufort, South Africa

\#Sharlotte Paulina Sehlapelo; B.Occupational Therapy (SU). https://orcid.org/0000-0002-1598-2858

Occupational Therapist, Van Velden Hospital, Tzaneen, South Africa

\#Marianne Breytenbach; B.Occupational Therapy (SU). https://orcid.org/0000-000 I-5569-0289

Occupational Therapist, St Barnabas Hospital, Libode, Eastern Cape, South Africa

\# B. Occupational Therapy students at the time of the study.

\section{DEAR EDITOR}

\section{INTRODUCTION}

Based on an on-going multi-international study by the International Expert Advisory Panel on Community Health and Transport $(\mathrm{I}-\mathrm{CHaT})^{\prime}$ a quantitative cross-sectional descriptive study was conducted in 2018. The study was approved by the Stellenbosch University Undergraduate Research Ethics and Health Research Committee. Ethics Clearance Number U I 7/ 10/053. Convenience sampling was used to recruit eligible participants from three population groups within Worcester. These three population groups constituted individuals from Innovation for the Blind (IFB) for visually impaired persons, National Institute for the Deaf (NID) for persons with hearing loss, as well as the Worcester Community Day Clinic (CDC) which serves the whole community. The CDC was used to recruit elderly people without any sensory impairments.

Community mobility refers to an individual's ability to move around in the community using various modes of transport such as taxis, buses, driving, cycling, or walking ${ }^{2}$. Walking in the context of this study included individuals who walked with and without the use of aids. Access to various modes of transport is fundamental for com- munity mobility which, in turn, plays a significant role in occupational engagement, personal and community living and self-care in older adults $^{2}$. In addition to referring to the way in which individuals pass through physical spaces and places, such as moving within the home to complete day-to-day tasks or commuting from home to work, mobility transition also refers to the modification of locomotive means over an individual's life span to best suit their physical abilities which change with ageing. Therefore, with ageing, mobility transition plays a fundamental role in maintaining community mobility.

Community mobility also contributes to giving an individual a sense of identity and assists in accessing health services. Understandably, a lack thereof is linked to feelings of loneliness, deteriorating health, and symptoms of depression ${ }^{2,3}$. Consequently, maintaining community mobility plays a substantial role in preserving a good quality of life as one advances into the late stages of adulthood. In a context with poor infrastructure, such as in South Africa, maintaining community mobility as one ages can often be a difficult task. Both the social development and public health systems are challenged with the important task of developing effective and efficient strate- 
gies to promote health and well-being in a growing and increasingly diverse ageing population.

A situational analysis done for the South African Plan of Action for Older Adults showed that older adults in rural areas especially, experience challenges to access health care services available to them. The main challenge identified was the lack of appropriate transport and information on the availability of transport services ${ }^{4}$. These findings motivated the researchers to investigate the forms of transport used by older adults in the Worcester area, a semi-rural agricultural town in Western Cape, South Africa, to access their out-of-home occupations. Unique to this study is the inclusion of older adults who present with various sensory impairments.

\section{METHOD}

Healthcare professionals from all three facilities were given a pre-defined eligibility criteria and assisted by the researchers in recruiting eligible participants. The recruited participants were screened using the World Health Organization Disability Assessment Schedule 2.0 (WHODAS 2.0 $)^{5}$ which has been standardised in both English and Afrikaans, to assess for difficulties experienced within the past month as a result of any impairment. Participants were considered eligible to continue with the study if they had provided informed consent, could mobilise independently without a wheelchair and if they obtained a disability score of less than $60 \%$ on the WHODAS 2.0. 58 participants took part in the study: 16 (28\%) from IFB, 20 (34\%) from NID and 22 (38\%) from CDC. All participants who were screened and eligible for the study proceeded to participate in a structured interview. The interview form used in the study included questions on demographic information, as well as aspects from a questionnaire on Accessing Places and Activities for Participation Outside Home for Older Adults (ACT-OUT) ${ }^{6}$. Specific aspects from the ACT-OUT interview form include modes of transport used - more than one mode could be selected, frequency used and satisfaction with these - and places of interest visited regularly. Interviews were conducted in English, Afrikaans and isiXhosa, the predominant languages in this context. South African Sign Language (SASL) interpreters were utilized to communicate with people with hearing impairments. The data were analysed using the technological tools namely SPSS version $24^{7}$ and STATA version $15^{8}$.

\section{RESULTS}

Results showed that the most frequently used modes of transport were walking with or without the use of aids (48\%), institutional transport (41\%), lifts from family members who drive vehicles $(28 \%)$, minibus taxi $(21 \%)$ and own vehicle (14\%). When considering the individual population groups, none of the IFB participants used their own vehicles, minibus taxis or lifts provided by family members with vehicles as transportation modes. Instead, $62 \%$ (10/16) of them used institutional transport and 56\% (9/16) used walking as modes of transport. From the NID participants $70 \%$ (14/20) utilised institutional transport and 60\% (12/20) walked to attend places of interest. Furthermore, $45 \%$ of NID (9/20) participants also relied on family members for transport, while $25 \%(5 / 20)$ relied on a minibus taxi and $5 \%(\mathrm{I} / 20)$ their own vehicle, to mobilise in the community. At the CDC, $50 \%(\mathrm{II} / 22)$ of participants used minibus taxis as transport, while $32 \%(7 / 22)$ relied frequently on family members or walking $27 \%$ (6/22). Additionally, I4\% (3/22) of CDC participants made use of their own vehicle while none used institutional transport.

The three most frequent modes of transport in this study were walking, transport provided by the institution and lifts provided by family members. The majority of those who walked were from the IFB and NID. Not only are both these institutions in central town, but participants from the IFB are also assisted by user-friendly adaptations like stud-patterned curb ramps and blind-crossings available in central town, while those from the NID have most of their places of interest located within the institution. Participants from CDC were impacted by age-related mobility disability, live on the outskirts of Worcester, and normally need to travel long distances to access their places of interest. This might explain the significant difference in percentages in walking as a mode of transport between CDC versus NID and IFB as we understand the additional physical demands of the former.

Participants from CDC compensate for the unavailability of institutional transport by using minibus taxis as the most readily available mode of transport in their community. With that being said, the obstacles with public transport faced by Worcester community-dwellers are similar to those highlighted by Ramachandran and D'Souza in a similar research study conducted in a rural setting in India ${ }^{2}$. These include high taxi costs and train stations being too far, all barriers to the successful travelling of the older adults. Albeit minibus taxis are not popular within the community, their use is not a matter of preference, but rather of limited transportation options as the commuters who make use of them indicated that they have never owned a driver's license or do not drive anymore. In addition, more convenient forms of transport such as Uber and buses are not available in the community, further restricting the use of public transport to minibus taxis. The prevalence of gang-related activity especially on the outskirts of Worcester appeared to have influenced the modes of transport participants used. Participants reported that they opted not to walk certain routes nor to use certain modes of transport at certain times out of fear, which predominantly resulted in participants not engaging in certain out-of-home occupations altogether.

Looking at lifts from family members, none of the IFB participants used this mode of transport, possibly because many of them have lived in the institution their entire lives and have never had to rely on family members, and rather make use of the institutional transport which is offered. While this finding can imply that IFB participants are the most independent of the three population groups it could also indicate they are best supported and that the other two population groups need additional support in order to attain the same level of independence.

\section{CONCLUSION}

From the findings, it is evident that walking, despite being physically demanding and difficult for the older adults, serves as a primary mode of transport. We therefore recommend that the local municipality and relevant facilities prioritise creating a safe and pedestrian-friendly environment for all older adults, in keeping with the policies. However, the question remains how the quality of life of a CDC member without institutional transport, who is not flexible to use minibus taxis and with no relative to rely on, is sustained? Institutions and communities alike should also take pride in providing transportation opportunities that are comfortable, affordable and user-friendly to their users. Institutions should broaden the variety of places that their older adults can visit using institutional transport. We also recommend a more in-depth qualitative study capturing the behavioural transition of older adults. 


\section{ACKNOWLEDGEMENTS}

The authors would like to thank Ms Tonya Esterhuizen, Dr Amy Slogrove and the Stellenbosch University Department of Occupational Therapy, the Ukwanda Centre for Rural Health, staff - and participants at NID, IFD and Worcester CDC for their contributions to the study.

\section{ROLE OF AUTHORS}

Asisipho Tsotsa, Rheta-Maria Smith, Tamsin Ballantyne, Isolde Burger, Sharlotte Sehlapelo and Marianne Breytenbach conducted the study as $4^{\text {th }}$ year occupational therapy students under the supervision of Catharina Duvenage and co-supervision of Lizette Swanepoel. Asisipho Tsotsa, Rheta-Maria Smith, Tamsin Ballantyne, Burger, Sharlotte Sehlapelo and Marianne Breytenbach wrote the first draft of the manuscript under the guidance of Kopane Dube. Kopane Dube coordinated the revisions of the manuscript. All the authors contributed to and approved the submitted version.

\section{REFERENCES}

I. Vaucher, P. et al. Development of the International Expert Advisory Panel on Community Health and Transport (I-CHaT) to coordinate research on transport mobility. Br. J. Occup. Ther. 8I, 245-246 (2018). https://doi.org/10.1 177/0308022617733389

2. Ramachandran, M. \& D'Souza, S. A. A Cross-Sectional Survey on Older Adults' Community Mobility in an Indian Metropolis. J. Cross.

Cult. Gerontol. 31, 19-33 (2016). https://doi.org//0.1007/s 10823-015-9276-7

3. Vanderschuren, M. J., Baufeldt, J. L. \& Phayane, S. Mobility barriers for older persons and people with Universal Design needs in South Africa. in 14th International Conference on Mobility and Transport for Elderly and Disabled Persons (2015). Researchgate.

4. Skweyiya, Z. SA Plan of Action for Older Persons. (2006) https://www.tafta.org.za/images/SAPlanofActiononAgeing.pdf

5. World Health Organization. WHO Disability Assessment Schedule (WHODAS 2.0). (2010). https://www.who.int/standards/classifications/international-classification-of-functioning-disability-and-health/ who-disability-assessment-schedule

6. Margot-Cattin, I. et al. Development of a questionnaire to evaluate out-of-home participation for people with dementia. Am. J. Occup. Ther. 73, I-10 (2019). https://doi.org/10.50 I4/ajot.2019.027I 44

7. IBM Corp. IBM SPSS Statistics for Windows. (2016).

8. StataCorp. Stata Statistical Software. (20I7). 